\title{
The Role of Color Doppler Ultrasound Arterial Mapping for Decision Making in the Treatment of Patients with Lower Extremity Peripheral Arterial Disease
}

\author{
Ali Babaei Jandaghi ${ }^{1}$, Zahra Mardanshahi ${ }^{1}$, Ahmad Alizadeh ${ }^{1}$, Iraj Baghi ${ }^{2}$, Hossein Hemmati ${ }^{3}$, \\ Narges Tabarzan Baboli ${ }^{4}$, Shabnam Alizadeh Arasi ${ }^{4}$, Amin Keshavarzzirak ${ }^{{ }^{*}}$ \\ ${ }^{1}$ Department of Radiology, Poursina Hospital, Guilan University of Medical Sciences, Rasht, Iran \\ ${ }^{2}$ Department of Vascular Surgery, Poursina Hospital, Guilan University of Medical Sciences, Rasht, Iran \\ ${ }^{3}$ Department of Vascular Surgery, Razi Hospital, Guilan University of Medical Sciences, Rasht, Iran \\ ${ }^{4}$ Department of Cardiology, Heshmat Hospital, Guilan University of Medical Sciences, Rasht, Iran \\ Email: *amin_keshavarzzirak@yahoo.com
}

Received August 9, 2013; revised August 31, 2013; accepted September 7, 2013

Copyright (C) 2013 Ali Babaei Jandaghi et al. This is an open access article distributed under the Creative Commons Attribution License, which permits unrestricted use, distribution, and reproduction in any medium, provided the original work is properly cited.

\begin{abstract}
Purpose: To assess the efficacy of color Doppler imaging for decision making in the treatment of patients with lower extremity peripheral arterial disease (PAD) compared to digital subtraction angiography (DSA). Materials and Methods: Color Doppler scan was done on patients suspected for lower limb PAD, a day prior to the DSA which was done by a vascular surgeon. Also, for the patients who were candidates for endovascular intervention based on the color Doppler arterial mapping results, endovascular interventions were performed at the same time if the DSA findings are correlated with the color Doppler map. The grading for evaluated segments was normal, insignificant stenosis $(<50 \%)$, hemodynamically significant stenosis $(\geq 50 \%)$ and occlusion. We yielded the diagnostic efficacy indices of Doppler for detecting arterial stenosis in each 18 different arterial segments below the renal arteries including, infrarenal aorta, common and external iliac, common femoral, superficial femoral (proximal, middle and distal segments), deep femoral, popliteal artery, tibioperoneal trunk, anterior and posterior tibial arteries (proximal, middle and distal segments) and peroneal artery (proximal and distal segments). Then, we yielded the kappa agreement between Doppler and DSA findings considering the grade of stenosis in 18 arterial segments separately. Results: Totally 115 lower extremities (2045 arterial segments) were evaluated in 90 patients [mean age: $60.8 \pm 8.9$ (range: 47 - 84 years old)] of which 68 (75.6\%) were men. The sensitivity of color Doppler for all arterial segments was $90 \%$ or higher except for common iliac artery, distal segment of superficial femoral artery and proximal segments of anterior and posterior tibialis and peroneal arteries. However, the specificity was $89 \%$ or higher, in all arterial segments. Kappa agreement was 0.72 or higher in all segments (All P-Values $<0.001$ ). Conclusion: This study suggests that considering excellent capability of color Doppler sonography in the evaluation of lower extremity arterial disease, color Doppler arterial mapping is sufficient for decision making in the treatment of these patients and can reduce the rate of diagnostic angiography.
\end{abstract}

Keywords: Lower Extremity; Peripheral Arterial Disease; Color Doppler Sonography; Arterial Mapping; Digital Subtraction Angiography

\section{Introduction}

Peripheral arterial disease (PAD) is a common problem in the elderly, especially among diabetic patients. When PAD limits the patients' lifestyle, diagnostic imaging is performed to characterize the number, length, level and severity of the lesions. Although Doppler ultrasonography is safe, relatively inexpensive, reproducible, a nonionizing method, non-invasive and widely available as an

\footnotetext{
"Corresponding author.
}

outpatient service, it is yet time consuming and operator dependent. On the other hand, digital subtraction angiography (DSA) provides easily visualized images of arterial tree. It can be used for endovascular interventions. However, it's of high cost, ionizing radiation, need for contrast agents and invasive nature, and makes this procedure unsuitable for screening or for follow-up purposes [1-4].

Color Doppler imaging is not only a morphological but also a functional study, providing information about 
both the vessel hemodynamic and wall.

Some studies have found that not only color Doppler could replace up to $97 \%$ of diagnostic arteriography of the lower limb [5] but also it could safely and accurately guide therapeutic vascular interventions [6], thus suggesting that DSA which has been considered by many as the gold standard, may no longer be regarded so.

Recent studies indicate that the sensitivity and specificity of an arterial duplex study and color flow imaging have increased significantly, making it a highly effective modality for non-invasive evaluation of PAD [7].

Current arterial duplex modalities offer significantly better anatomic evaluation for lesions in PAD patients [8-12]. In some recent studies it has shown that there are good concordances between arterial duplex studies and DSA in aorto-iliac and femoro-popliteal disease, and it's fair to poor concordances in popliteal and infrapopliteal disease [9-12].

In recent studies, it has shown that color Doppler sonography is useful in planning treatment in patients with PAD $[13,14]$.

Our aim in this study was to determine whether the color Doppler arterial mapping would be useful in treatment planning in patients with lower extremity peripheral arterial disease (PAD) by providing an image of the arterial tree for the clinician.

\section{Materials and Methods}

Totally 115 lower extremities (2045 arterial segments) were evaluated in 90 patients suspected for lower limb PAD [mean age: $60.8 \pm 8.9$ (range: $47-84$ years old)] of which 68 (75.6\%) were men. Color and spectral Doppler scan was done a day prior to the DSA which was done by a vascular surgeon. All subjects were examined with B-mode, color and duplex Doppler US using a 2 - 5-MHz convex array transducer for intra-abdominal and pelvic arterial evaluation, and a $7-12-\mathrm{MHz}$ linear array trans- ducer for infra-inguinal arterial assessment (SONIX OP). The distal portion of superficial femoral artery in the Hunter area was also evaluated with the $2-5-\mathrm{MHz}$ convex array probe.

Spectral analysis was recorded for at least two to three cycles of the waveform and the Doppler angle was set at less than $60^{\circ}$. Measurements were performed on a frozen spectral strip. The values of the maximum peak systolic velocity (Vmax, in $\mathrm{cm} / \mathrm{s}$ ) were obtained in all subjects. The grading for evaluated segments was normal, insignificant stenosis $(<50 \%)$, hemodynamically significant stenosis $(\geq 50 \%)$, and occlusion (Figure 1). We yielded the diagnostic efficacy indices of Doppler for detecting arterial stenosis in each 18 different arterial segments below the renal arteries including, infrarenal aorta, common and external iliac, common femoral, deep femoral, superficial femoral (proximal, middle and distal segments), popliteal artery, tibioperoneal trunk, anterior and posterior tibial arteries (proximal, middle and distal segments) and peroneal artery (proximal and distal segments). The information from the color Doppler study was entered on a data sheet containing the diagrams of the lower limbs arteries (Figure 2). Then, we yielded the kappa agreement between Doppler and DSA findings considering the grade of stenosis in 18 arterial segments separately.

For the patients who were candidates for endovascular intervention based on the color Doppler arterial mapping results, endovascular interventions were performed at the same time if the DSA findings correlated with the color Doppler map. However, diagnostic DSA angiography was also performed on those patients who were candidates for vascular surgery based on color Doppler mapping.

Written informed consent was obtained from all participants.

All procedures were in accordance with the ethical standards of the responsible committee on human ex-
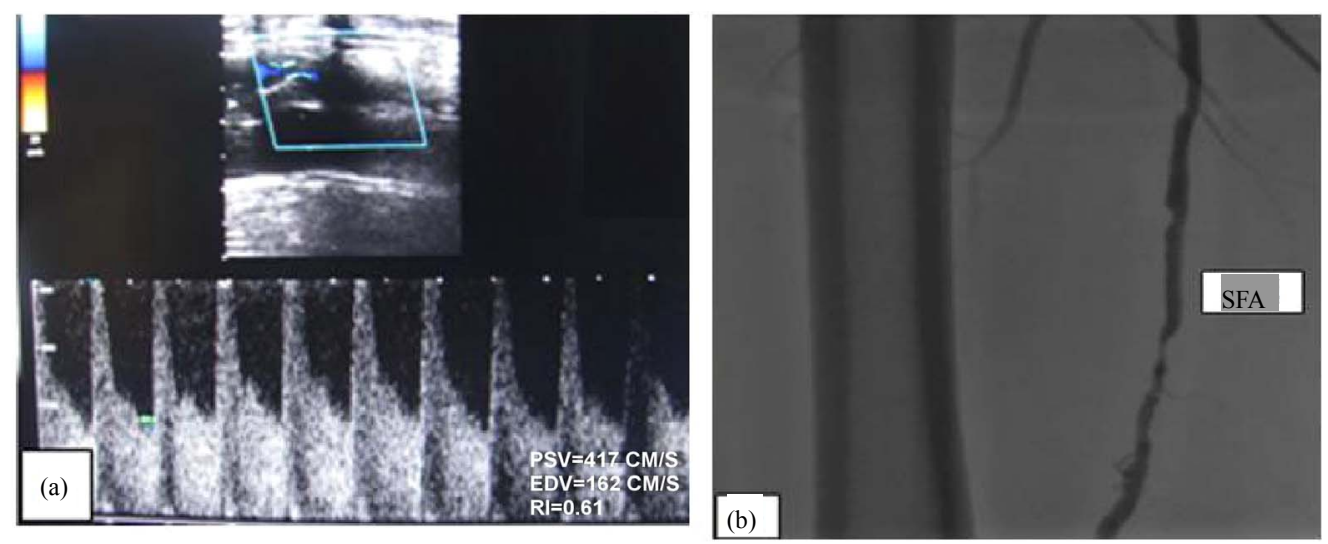

Figure 1. Significant stenosis in superficial femoral artery. (a) Increased peak systolic velocity (PSV) in the proximal segment of superficial femoral artery (more than $417 \mathrm{~cm} / \mathrm{s}$ ) indicating significant stenosis. (b) Corresponding DSA, indicating significant stenosis in superficial femoral artery. 
perimentation (institutional and national) and with the Helsinki Declaration of 1975, as revised in 2000 [15].

\section{Results}

In the infra renal aorta, external iliac, common femoral, deep femoral, superficial femoral (proximal and middle segments), popliteal, tibioperoneal trunk, anterior tibialis (middle and distal segments), posterior tibialis (middle and distal segments) and peroneal (distal segment) arteries, color Doppler showed a sensitivity greater than $90 \%$ in diagnosing stenosis and occlusion. On the other hand, in common iliac, distal segment of superficial femoral and proximal segments of anterior and posterior tibialis and peroneal arteries, sensitivity was $82 \%$ to $90 \%$. Also, in all segments, the specificity was equal or greater than 89\% (Table 1).

For all arterial segments, the kappa values were equal or greater than 0.72 (All P Values lower than 0.0001). (Table 2)

\section{Discussion}

In recent years, the role of duplex scanning as a prepro-

Table 1. Diagnostic indices of color Doppler sonography.

\begin{tabular}{|c|c|c|c|c|c|c|c|}
\hline & Sen. & Spec. & PPV & NPV & PLR & NLR & Efficacy \\
\hline $\begin{array}{l}\text { Infra renal aorta } \\
\quad(\mathrm{C} 195 \%)\end{array}$ & $\begin{array}{l}100 \% \\
(----)\end{array}$ & $\begin{array}{l}100 \% \\
(----)\end{array}$ & $\begin{array}{l}100 \% \\
(----)\end{array}$ & $\begin{array}{l}100 \% \\
(----)\end{array}$ & (--- & (---- & $\begin{array}{l}100 \% \\
(----)\end{array}$ \\
\hline $\begin{array}{c}\text { Common iliac artery } \\
\text { (C195\%) }\end{array}$ & $\begin{array}{c}88.9 \% \\
(74 \%-100 \%)\end{array}$ & $\begin{array}{c}95.9 \% \\
(92 \%-99 \%)\end{array}$ & $\begin{array}{c}80 \% \\
(62.5 \%-97.5 \%)\end{array}$ & $\begin{array}{c}97.9 \% \\
(95 \%-100 \%)\end{array}$ & $\begin{array}{c}21.7 \% \\
(14.2 \%-29.2 \%)\end{array}$ & $\begin{array}{c}8.6 \% \\
(3.5 \%-13.7 \%)\end{array}$ & $\begin{array}{c}94.8 \% \\
(90.7 \%-98.9 \%)\end{array}$ \\
\hline $\begin{array}{c}\text { External iliac artery } \\
\text { (CI95\%) }\end{array}$ & $\begin{array}{c}93.8 \% \\
(82 \%-100 \%)\end{array}$ & $\begin{array}{l}100 \% \\
(----)\end{array}$ & $\begin{array}{l}100 \% \\
(----)\end{array}$ & $\begin{array}{c}99 \% \\
(97 \%-100 \%)\end{array}$ & (---- & $\begin{array}{c}16.1 \% \\
(9.4 \%-22.8 \%)\end{array}$ & $\begin{array}{c}99 \% \\
(97.2 \%-100 \%)\end{array}$ \\
\hline $\begin{array}{l}\text { Common femoral } \\
\text { artery }(\mathrm{CI} 95 \%)\end{array}$ & $\begin{array}{l}100 \% \\
(----)\end{array}$ & $\begin{array}{l}100 \% \\
(----)\end{array}$ & $\begin{array}{l}100 \% \\
(----)\end{array}$ & $\begin{array}{l}100 \% \\
(----)\end{array}$ & (---- & (----- & $\begin{array}{l}100 \% \\
(----)\end{array}$ \\
\hline $\begin{array}{l}\text { Deep femoral artery } \\
(\text { (CI95\%) }\end{array}$ & $\begin{array}{c}97.1 \% \\
(91.5 \%-100 \%)\end{array}$ & $\begin{array}{c}97.5 \% \\
(94.1 \%-100 \%)\end{array}$ & $\begin{array}{c}94.4 \% \\
(86.9 \%-100 \%)\end{array}$ & $\begin{array}{c}98.7 \% \\
(96.2 \%-100 \%)\end{array}$ & $\begin{array}{c}38.8 \% \\
(29.9 \%-47.7 \%)\end{array}$ & $\begin{array}{c}33.6 \% \\
(25 \%-42.2 \%)\end{array}$ & $\begin{array}{c}97.3 \% \\
(94.3 \%-100 \%)\end{array}$ \\
\hline $\begin{array}{c}\text { Superficial femoral } \\
\text { artery } 1 / 3 \text { proximal } \\
\text { (CI95\%) }\end{array}$ & $\begin{array}{c}97.1 \% \\
(91.5 \%-100 \%)\end{array}$ & $\begin{array}{c}97.5 \% \\
(94.1 \%-100 \%)\end{array}$ & $\begin{array}{c}94.4 \% \\
(86.9 \%-100 \%)\end{array}$ & $\begin{array}{c}98.7 \% \\
(96.2 \%-100 \%)\end{array}$ & $\begin{array}{c}38.8 \% \\
(29.9 \%-47.7 \%)\end{array}$ & $\begin{array}{c}33.6 \% \\
(25 \%-42.2 \%)\end{array}$ & $\begin{array}{c}97.3 \% \\
(94.3 \%-100 \%)\end{array}$ \\
\hline $\begin{array}{c}\text { Superficial femoral } \\
\text { artery } 1 / 3 \text { middle } \\
(\text { CI } 95 \%)\end{array}$ & $\begin{array}{c}95.9 \% \\
(90.3 \%-100 \%)\end{array}$ & $\begin{array}{c}97 \% \\
(92.9 \%-100 \%)\end{array}$ & $\begin{array}{c}95.9 \% \\
(90.3 \%-100 \%)\end{array}$ & $\begin{array}{c}97 \% \\
(92.9 \%-100 \%)\end{array}$ & $\begin{array}{c}31.9 \% \\
(23.4 \%-40.4 \%)\end{array}$ & $\begin{array}{c}23.6 \% \\
(15.8 \%-31.4 \%)\end{array}$ & $\begin{array}{c}96.5 \% \\
(93.1 \%-99 \%)\end{array}$ \\
\hline $\begin{array}{l}\text { Superficial femoral } \\
\text { artery } 1 / 3 \text { distal } \\
(\mathrm{CI} 95 \%)\end{array}$ & $\begin{array}{c}87.1 \% \\
(75.3 \%-98.9 \%)\end{array}$ & $\begin{array}{c}89.3 \% \\
(82.7 \%-95.9 \%)\end{array}$ & $\begin{array}{c}75 \% \\
(60.9 \%-89.1 \%)\end{array}$ & $\begin{array}{c}94.9 \% \\
(90 \%-99.8 \%)\end{array}$ & $\begin{array}{c}81.4 \% \\
(74.3 \%-88.5 \%)\end{array}$ & $\begin{array}{c}69.2 \% \\
(60.8 \%-77.6 \%)\end{array}$ & $\begin{array}{c}88.7 \% \\
(82.9 \%-945 \%)\end{array}$ \\
\hline $\begin{array}{l}\text { Popliteal artery } \\
\text { (CI95\%) }\end{array}$ & $\begin{array}{c}94.4 \% \\
(83 \%-100 \%)\end{array}$ & $\begin{array}{c}96.9 \% \\
(93.5 \%-100 \%)\end{array}$ & $\begin{array}{c}85 \% \\
(69.4 \%-100 \%)\end{array}$ & $\begin{array}{c}98.9 \% \\
(96.8 \%-100 \%)\end{array}$ & $\begin{array}{c}30.4 \% \\
(22 \%-38.8 \%)\end{array}$ & $\begin{array}{c}17.3 \% \\
(10.4 \%-24.2 \%)\end{array}$ & $\begin{array}{c}96.5 \% \\
(93.1 \%-99.9 \%)\end{array}$ \\
\hline $\begin{array}{l}\text { Tibioproneal trunk } \\
\text { (CI95\%) }\end{array}$ & $\begin{array}{c}90 \% \\
(76.9 \%-100 \%)\end{array}$ & $\begin{array}{c}96.8 \% \\
(93.3 \%-100 \%)\end{array}$ & $\begin{array}{c}85.7 \% \\
(70.7 \%-100 \%)\end{array}$ & $\begin{array}{c}97.9 \% \\
(95 \%-100 \%)\end{array}$ & $\begin{array}{c}28.1 \% \\
(19.9 \%-36.3 \%)\end{array}$ & $\begin{array}{c}9.7 \% \\
(4.3 \%-15.1 \%)\end{array}$ & $\begin{array}{c}95.6 \% \\
(91.9 \%-99.3 \%)\end{array}$ \\
\hline $\begin{array}{l}\text { Anterior tibial artery } \\
\text { proximal (CI95\%) }\end{array}$ & $\begin{array}{c}85.3 \% \\
(73.4 \%-97.2 \%)\end{array}$ & $\begin{array}{c}92.6 \% \\
(86.9 \%-98.3 \%)\end{array}$ & $\begin{array}{c}82.9 \% \\
(70.4 \%-95.4 \%)\end{array}$ & $\begin{array}{c}93.8 \% \\
(88.5 \%-99.1 \%)\end{array}$ & $\begin{array}{c}11.5 \% \\
(5.7 \%-17.3 \%)\end{array}$ & $\begin{array}{c}63 \% \\
(1.9 \%-10.7 \%)\end{array}$ & $\begin{array}{c}90 \% \\
(84.5 \%-95.5 \%)\end{array}$ \\
\hline $\begin{array}{l}\text { Anterior tibial artery } \\
\text { middle (CI95\%) }\end{array}$ & $\begin{array}{c}93.3 \% \\
(84.4 \%-100 \%)\end{array}$ & $\begin{array}{c}96.5 \% \\
(92.6 \%-100 \%)\end{array}$ & $\begin{array}{c}90.3 \% \\
(79.9 \%-100 \%)\end{array}$ & $\begin{array}{c}97.6 \% \\
(94.3 \%-100 \%)\end{array}$ & $\begin{array}{c}26.7 \% \\
(18.6 \%-34.6 \%)\end{array}$ & $\begin{array}{c}14.4 \% \\
(8 \%-20.8 \%)\end{array}$ & $\begin{array}{c}90 \% \\
(84.5 \%-95.5 \%)\end{array}$ \\
\hline $\begin{array}{l}\text { Anterior tibial artery } \\
\quad \text { distal }(\mathrm{CI} 95 \%)\end{array}$ & $\begin{array}{l}100 \% \\
(---)\end{array}$ & $\begin{array}{l}100 \% \\
(---)\end{array}$ & $\begin{array}{l}100 \% \\
(----)\end{array}$ & $\begin{array}{l}100 \% \\
(----)\end{array}$ & $(---)$ & (----- & $\begin{array}{l}100 \% \\
(----)\end{array}$ \\
\hline $\begin{array}{l}\text { Posterior tibial artery } \\
\text { proximal (CI95\%) }\end{array}$ & $\begin{array}{c}89.8 \% \\
(81.3 \%-98.3 \%)\end{array}$ & $\begin{array}{c}90.9 \% \\
(84 \%-97.8 \%)\end{array}$ & $\begin{array}{c}88 \% \\
(79 \%-97 \%)\end{array}$ & $\begin{array}{c}92.3 \% \\
(85.8 \%-98.8 \%)\end{array}$ & $\begin{array}{c}9.8 \% \\
(4.4 \%-15.2 \%)\end{array}$ & $\begin{array}{c}8.9 \% \\
(3.7 \%-14.1 \%)\end{array}$ & $\begin{array}{c}90 \% \\
(84.5 \%-95.5 \%)\end{array}$ \\
\hline $\begin{array}{l}\text { Posterior tibial artery } \\
\text { middle }(\mathrm{CI} 95 \%)\end{array}$ & $\begin{array}{c}92.2 \% \\
(84.8 \%-99.6 \%)\end{array}$ & $\begin{array}{c}95.3 \% \\
(90.1 \%-100 \%)\end{array}$ & $\begin{array}{c}94 \% \\
(87.4 \%-100 \%)\end{array}$ & $\begin{array}{c}93.8 \% \\
(87.9 \%-99.7 \%)\end{array}$ & $\begin{array}{c}19.6 \% \\
(12.3 \%-26.9 \%)\end{array}$ & $\begin{array}{c}12.2 \% \\
(6.2 \%-18.2 \%)\end{array}$ & $\begin{array}{c}93.9 \% \\
(89.5 \%-98.3 \%)\end{array}$ \\
\hline $\begin{array}{l}\text { Posterior tibial artery } \\
\text { (C195\%) }\end{array}$ & $\begin{array}{c}96.4 \% \\
(89.5 \%-100 \%)\end{array}$ & $\begin{array}{l}100 \% \\
(----)\end{array}$ & $\begin{array}{l}100 \% \\
(----)\end{array}$ & $\begin{array}{c}98.9 \% \\
(96.7 \%-100 \%)\end{array}$ & $\begin{array}{l}100 \% \\
(----)\end{array}$ & $\begin{array}{c}17.9 \% \\
(10.9 \%-24.9 \%)\end{array}$ & $\begin{array}{c}96.5 \% \\
(93.1 \%-99.9 \%)\end{array}$ \\
\hline $\begin{array}{l}\text { Peroneal artery } \\
\text { proximal (CI95\%) }\end{array}$ & $\begin{array}{c}82.8 \% \\
(69.1 \%-96.5 \%)\end{array}$ & $\begin{array}{c}93 \% \\
(87.6 \%-98.4 \%)\end{array}$ & $\begin{array}{c}80 \% \\
(65.7 \%-94.3 \%)\end{array}$ & $\begin{array}{c}94.1 \% \\
(89.1 \%-99.1 \%)\end{array}$ & $\begin{array}{c}11.8 \% \\
(5.9 \%-17.7 \%)\end{array}$ & $\begin{array}{c}13.9 \% \\
(7.6 \%-20.2 \%)\end{array}$ & $\begin{array}{c}90 \% \\
(84.5 \%-95.5 \%)\end{array}$ \\
\hline $\begin{array}{l}\text { Peroneal artery distal } \\
(\mathrm{C} 195 \%)\end{array}$ & $\begin{array}{c}92.9 \% \\
(83.4 \%-100 \%)\end{array}$ & $\begin{array}{c}95.4 \% \\
(91 \%-99.8 \%)\end{array}$ & $\begin{array}{c}86.7 \% \\
(74.5 \%-98.9 \%)\end{array}$ & $\begin{array}{c}97.6 \% \\
(94.3 \%-100 \%)\end{array}$ & $\begin{array}{c}20.2 \% \\
(12.9 \%-27.5 \%)\end{array}$ & $\begin{array}{c}13.4 \% \\
(7.2 \%-19.6 \%)\end{array}$ & $\begin{array}{c}94.7 \% \\
(90.6 \%-98.8 \%)\end{array}$ \\
\hline
\end{tabular}

Sen: Sensitivity, Spec: Specificity, PPV: Positive Predictive Value, NPV: Negative Predictive Value, PLR: Positive Likelihood Ratio, NLR: Negative Likelihood Ratio. 
Table 2. The kappa agreements for all arterial segments.

\begin{tabular}{|c|c|c|}
\hline & Kappa & P-value \\
\hline Infra renal aorta & 1 & $\mathrm{P}<0.0001$ \\
\hline Common iliac artery & 0.811 & $\mathrm{P}<0.0001$ \\
\hline External iliac artery & 0.963 & $\mathrm{P}<0.0001$ \\
\hline Common femoral artery & 1 & $\mathrm{P}<0.0001$ \\
\hline Deep femoral artery & 0.939 & $\mathrm{P}<0.0001$ \\
\hline Superficial femoral artery $1 / 3$ proximal & 0.939 & $\mathrm{P}<0.0001$ \\
\hline Superficial femoral artery $1 / 3$ middle & 0.929 & $\mathrm{P}<0.0001$ \\
\hline Superficial femoral artery $1 / 3$ distal & 0.727 & $\mathrm{P}<0.0001$ \\
\hline Popliteal artery & 0.874 & $\mathrm{P}<0.0001$ \\
\hline Tibioproneal trunk & 0.852 & $\mathrm{P}<0.0001$ \\
\hline Anterior tibial artery proximal & 0.772 & $\mathrm{P}<0.0001$ \\
\hline Anterior tibial artery middle & 0.888 & $\mathrm{P}<0.0001$ \\
\hline Anterior tibial artery distal & 1 & $\mathrm{P}<0.0001$ \\
\hline posterior tibial artery proximal & 0.805 & $\mathrm{P}<0.0001$ \\
\hline posterior tibial artery middle & 0.876 & $\mathrm{P}<0.0001$ \\
\hline posterior tibial artery & 0.976 & $\mathrm{P}<0.0001$ \\
\hline Peroneal artery proximal & 0.749 & $\mathrm{P}<0.0001$ \\
\hline Peroneal artery distal & 0.862 & $\mathrm{P}<0.0001$ \\
\hline
\end{tabular}

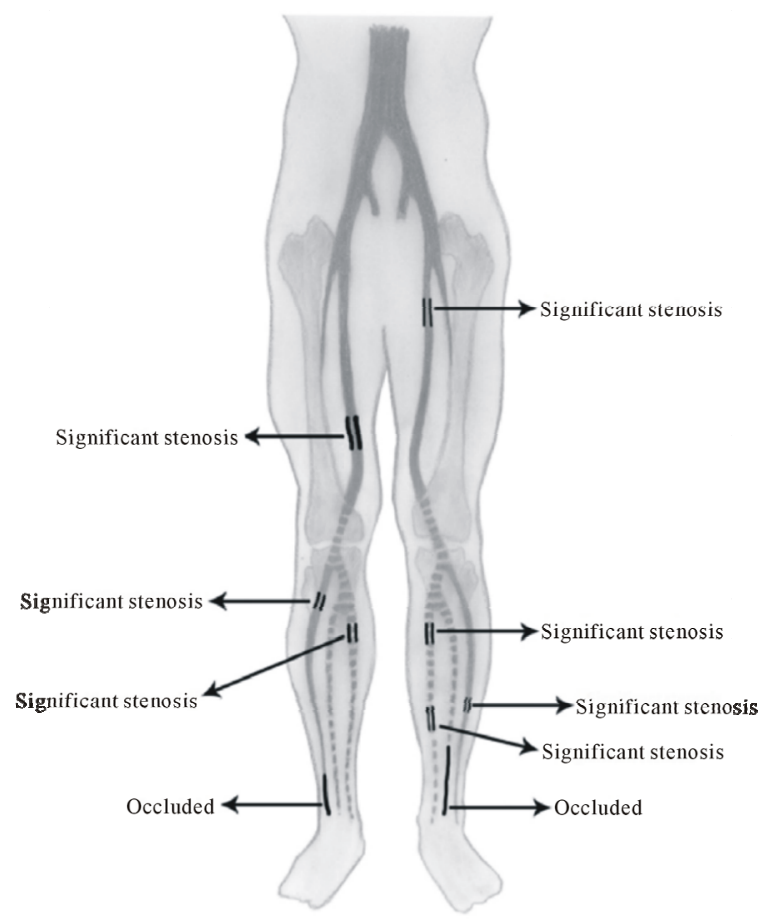

Figure 2. Color and Spectral Doppler ultrasound arterial mapping.

cedural diagnostic imaging for detecting and grading occlusive disease of the lower limbs has been under dis- cussion comparing with angiography as a "gold standard" $[13,14,16,17]$. The ultimate purpose of the diagnostic tools is not only limited to draw an accurate anatomic arterial mapping but also to establish an appropriate therapeutic strategy, either surgical or endovascular [16]. In these literatures, there is increasing evidence to indicate the possibility that Duplex scanning should replace angiography in many patients for the therapeutic planning $[13,14,16,17]$ especially in high risk patients with diabetes, renal failure or contrast agent allergy $[18,19]$.

In the present study, to evaluate the efficacy of duplex scanning in detecting the lower extremity arterial disease, 18 arterial segments (totally 2045) were evaluated in details and the arterial mapping of arterial beds was drawn. To our knowledge, in the previous studies $[7,8,13]$ such a segmental evaluation had not been done with this detail. Good diagnostic agreement $(\mathrm{k} \geq 0.72 ; \mathrm{P}<0.0001)$ was achieved in the all arterial segments. Excellent agreement was achieved in the infra-renal aorta, Common femoral artery and anterior tibial artery distal segments $(\mathrm{k}=1 ; \mathrm{P}<0.0001)$.

The study by Favaretto et al., showed poor agreement in infrapopliteal districts, with a low sensitivity and high specificity in detecting significant stenosis or occlusions [8]. Also Fontcuberta et al., showed lower values for sensitivity, specificity, positive predictive value and 
negative predictive value in tibial arteries than for other sectors [13]. In our study, good agreement was achieved in all below the knee arterial segments $(\mathrm{k} \geq 0.75 ; \mathrm{P}<$ $0.0001)$; however, lower agreement was noted for proximal segments of anterior tibialis and peroneal arteries ( $\mathrm{k}=0.77$ and 0.75 , respectively). Also, distal portion of SFA in Hunter canal was difficult to be evaluated. So, we used convex probe to overcome the poor view of this arterial segment. Nevertheless, the lowest agreement $(\mathrm{k}=$ 0.72 ) in our study was related to this segment. Although duplex scanning has lower sensitivity and specificity in distal segment of superficial femoral and proximal segments of anterior and posterior tibialis and peroneal arteries (Table 1), it is effective for drawing arterial mapping and further clinical decision making.

The arterial mapping in our study helped our vascular surgeon to be ready for possible interventional procedures such as need for stenting or angioplasty at the same time of diagnostic DSA angiography. This resulted in less patient's costs and necessity for second interventional angiography. Moreover, the patients were satisfied by one step intervention.

\section{Conclusion}

In conclusion, this study suggests that considering excellent capability of color Doppler sonography in the evaluation of lower extremity arterial disease, color Doppler arterial mapping is sufficient for decision making in treatment of these patients and can reduce the rate of diagnostic angiography.

\section{REFERENCES}

[1] K. A. Jager, D. J. Phillips, R. L. Martin, et al., "Noninvasive Mapping of Lower Limb Arterial Lesions," Ultrasound in Medicine and Biology, Vol. 11, No. 3, 1985, pp. 515-521. http://dx.doi.org/10.1016/0301-5629(85)90164-4

[2] T. R. Kohler, D. R. Nance, M. M. Cramer, et al., "Duplex Scanning for Diagnosis of Aortoiliac and Femoropopliteal Disease: A Prospective Study," Circulation, Vol. 76, No. 5, 1987, pp. 1074-1080.

http://dx.doi.org/10.1161/01.CIR.76.5.1074

[3] B. L. Thiele and D. E. Strandness Jr., "Accuracy of Angiographic Quantification of Peripheral Atherosclerosis," Progress in Cardiovascular Diseases, 1983, Vol. 26, No. 3, pp. 223-236.

http://dx.doi.org/10.1016/0033-0620(83)90007-5

[4] H. B. Slot, L. Strijbosch and J. M. Greep, "Interobserver Variability in Single-Plane Aortography," Surgery, Vol. 90, No. 3, 1981, pp. 497-503.

[5] M. Pemberton, S. Nydahl, T. Hartshorne, et al., "Can Lower Limb Vascular Reconstruction Be Based on Color Duplex Imaging Alone?" European Journal of Vascular \& Endovascular Surgery, Vol. 12, No. 4, 1996, pp. 452454. http://dx.doi.org/10.1016/S1078-5884(96)80013-X
[6] B. H. Elsman, D. A. Legemate, F. H. van der Heijden, et al., "Impact of Ultrasonographic Duplex Scanning on Therapeutic Decision Making in Lower-Limb Arterial Disease," British Journal of Surgery, Vol. 82, No. 5, 1995 , pp. 630-633.

[7] C. G. Koshy, B. R. Chacko and S. N. Keshava, "Diagnostic Accuracy of Color Doppler Imaging in the Evaluation of Peripheral Arterial Disease as Compared to Digital Subtraction Angiography," Vascular Disease Management, Vol. 6, No. 1, 2009, pp. 2-9.

[8] E. Favaretto, C. Pili, A. Amato, et al., "Analysis of Agreement between Duplex Ultrasound Scanning and Arteriography in Patients with Lower Limb Artery Disease," Journal of Cardiovascular Medicine, Vol. 8, No. 5, 2007, pp. 337-341. http://dx.doi.org/10.2459/01.JCM.0000268124.51543.b2

[9] A. Krnic, N. Vucic and Z. Sucic, "Duplex Scanning Compared with Intra-Arterial Angiography in Diagnosing Peripheral Arterial Disease: Three Analytical Approaches," Vasa, Vol. 35, No. 2, 2006, pp. 86-91. http://dx.doi.org/10.1024/0301-1526.35.2.86

[10] S. Aly, K. Sommerville, M. Adiseshiah, et al., "Comparison of Duplex Imaging and Arteriography in the Evaluation of Lower Limb Arteries," British Journal of Surgery, Vol. 85, No. 8, 1998, pp. 1099-1102. http://dx.doi.org/10.1046/j.1365-2168.1998.00786.x

[11] T. Leiner, A. G. Kessels, P. J. Nelemans, et al., "Peripheral Arterial Disease: Comparison of Color Duplex US and Contrast-Enhanced MR Angiography for Diagnosis," Radiology, Vol. 235, 2005, pp. 699-708. http://dx.doi.org/10.1148/radiol.2352040089

[12] J. F. Polak, M. I. Karmel, J. A. Mannick, et al., "Determination of the Extent of Lower-Extremity Peripheral Arterial Disease with Color-Assisted Duplex Sonography: Comparison with Angiography," American Journal of Roentgenology, Vol. 155, No. 5, 1990, pp. 1085-1089. http://dx.doi.org/10.2214/ajr.155.5.2120939

[13] J. Fontcuberta, A. Flores, A. Orgaz, M. Doblas, J. Gil, I. Leal, R. Rodriguez, J. Maria and M. Dolores, "Reliability of Preoperative Duplex Scanning in Designing a Therapeutic Strategy for Chronic Lower Limb Ischemia," Annals of Vascular Surgery, Vol. 23, No. 5, 2009, pp. $577-$ 582. http://dx.doi.org/10.1016/j.avsg.2008.07.011

[14] G. K. Chiramel, R. C. Binita, N. K. Shyamkumar, S. Edwin and A. Sunil, "Decision Making in the Treatment of Peripheral Arterial Disease-A Single-Institution Comparative Study Using Information from Color Doppler and Digital Subtraction Angiogram Studies," Indian Journal of Radiology and Imaging, Vol. 21, No. 4, 2011, pp. 294-297. http://dx.doi.org/10.4103/0971-3026.90694

[15] World Medical Association, "Declaration of HelsinkiEthical Principles for Medical Research Involving Human Subjects," 2012.

http://www.wma.net/en/30publications/10policies/b3/inde x.html

[16] A.-M. Löfberg, S. Karacagil, A. Hellberg, A. Boström, C. Ljungman and G. Östholm, "The Role of Duplex Scanning in the Selection of Patients with Critical Lower-Limb Ischemia for Infrainguinal Percutaneous Transluminal 
Angioplasty," CardioVascular and Interventional Radiology, Vol. 24, No. 4, 2001, pp. 229-232.

http://dx.doi.org/10.1007/s00270-001-1786-7

[17] J. A. Grassbaugh, P. R. Nelson, E. M. Rzucidlo, M. L. Schermerhorn, M. F. Filinger, R. J. Powell, et al., "Blinded Comparison of Preoperative Duplex Ultrasound Scanning and Contrast Arteriography for Planning Revascularization at the Level of Tibia," Journal of Vascular Surgery, Vol. 37, No. 6, 2003, pp. 1186-1190. http://dx.doi.org/10.1016/S0741-5214(03)00328-8

[18] J. Q. Alexander, S. M. Leos and S. G. Katz, "Is Duplex
Ultrasonography an Effective Single Modality for the Preoperative Evaluation of Peripheral Vascular Disease?" American Surgeon, Vol. 68, No. 12, 2002, pp. 1107-1110.

[19] E. Ascher, A. Hingorani, N. Markevich, W. Yorkovich, R. H. Shutzer, T. Jacob, et al., "Role of Duplex Arteriography as the Sole Preoperative Imaging Modality Prior to Lower Extremity Revascularization Surgery in Diabetic and Renal Patients," Annals of Vascular Surgery, Vol. 18, No. 4, 2004, pp. 433-439. http://dx.doi.org/10.1007/s10016-004-0058-x 